\title{
Gene therapy with adenovirus-mediated myocardial transfer of vascular endothelial growth factor 121 improves cardiac performance in a pacing model of congestive heart failure
}

\author{
Eros Leotta, MD \\ Gerald Patejunas, $\mathrm{PhD}^{\mathrm{a}}$ \\ Glenn Murphy, MDa \\ Joseph Szokol, MD \\ Leslie McGregor, BS ${ }^{\mathrm{a}}$ \\ JoAnn Carbray, BS ${ }^{\mathrm{a}}$ \\ Adam Hamawy, $\mathrm{MD}^{\mathrm{b}}$ \\ David Winchester, MD \\ Neil Hackett, $\mathrm{PhD}^{\mathrm{b}}$ \\ Ronald Crystal, MD \\ Todd Rosengart, MD
}

From Evanston Northwestern Healthcare, ${ }^{\mathrm{a}}$ Evanston, Ill, and the Institute of Genetic Medicine, ${ }^{\mathrm{b}}$ Weill Medical College of Cornell University, New York, NY.

Presented in part at the Seventy-third Annual Meeting of the American Heart Association, November 2000, New Orleans, La.

This work was supported in part by grants from the Jeffry M. and Barbara Picower Foundation, Palm Beach, Fla; National Institutes of Health grants RO1 HL 57318 and RO1 HL6698-01; Evanston Northwestern Healthcare Research Institute, Evanston, Ill; and GenVec, Inc, Gaithersburg, $\mathrm{Md}$

Received for publication June 18, 2001; revisions requested Aug 2, 2001; revisions received Aug 20, 2001; accepted for publication Oct 2, 2001.

Address for reprints: Todd K. Rosengart, MD, Evanston Hospital, Division of Cardiothoracic Surgery, Burch 100, 2650 Ridge Ave, Evanston, IL 60201 (E-mail: trosengart@enh.org).

J Thorac Cardiovasc Surg 2002;123: $1101-13$

Copyright $\odot 2002$ by The American Association for Thoracic Surgery

$0022-5223 / 2002 \$ 35.00+0 \quad \mathbf{1 2 / 1 / 1 2 1 0 4 4}$ doi: $10.1067 / \mathrm{mtc} .2002 .121044$
Background: Myocardial ischemia is the most common cause of congestive heart failure. Angiogenic therapy has recently been demonstrated to enhance myocardial perfusion in the ischemic setting. We therefore hypothesized that administration of adenovirus encoding for vascular endothelial growth factor could be used to enhance myocardial function in a pacing-induced model of heart failure.

Methods: Yorkshire swine underwent a left thoracotomy with placement of a ventricular epicardial pacing system. Animals received adenovirus coding either for the 121-amino-acid isoform of vascular endothelial growth factor $\left(\mathrm{Ad}_{\mathrm{CU}} \mathrm{VEGF} \mathrm{V} 1.1\right.$ group, $\mathrm{n}=8$ ) or a null vector coding for no genes (AdNull group, $\mathrm{n}=8$ ). The adenovirus was administered in the left ventricular free wall as 10 transepicardial injections of $100 \mu \mathrm{L}$ each (total dose of $10^{11}$ particle units). After a 1-week recovery period, animals were paced at a rate of 230 beats/min for 7 days to induce heart failure. Transthoracic echocardiographic and sonomicrometric measurements were performed before pacing (baseline), on termination of pacing (day 0), and then weekly for 3 weeks.

Results: The fractional area change was significantly decreased in AdNull animals at day 0 after pacing compared with the $\mathrm{Ad}_{\mathrm{CU}}$ VEGF121.1 animals $(29 \% \pm 14 \%$ vs $46 \% \pm 8 \%, P=.02)$. The fractional area change recovered to baseline values within 7 days in the $\mathrm{Ad}_{\mathrm{CU}}$ VEGF121.1 animals $(62 \% \pm 7 \%)$ but remained significantly impaired in the AdNull group compared with that in the $\mathrm{Ad}_{\mathrm{CU}}$ VEGF121.1 animals up to day $21(P=.04)$. Similarly, fractional wall thickening demonstrated a decrease at day 0 after pacing that was greater $(P<.05)$ in the AdNull group compared with that in the $\mathrm{Ad}_{\mathrm{CU}}$ VEGF121.1 group in 5 of 6 segments. Fractional wall thickening returned to levels approximating prepacing values in all segments within 7 days in the $\mathrm{Ad}_{\mathrm{CU}}$ VEGF121.1 group but remained significantly impaired compared with prepacing fractional wall thickening $(P<.05)$ in the AdNull group in 5 of 6 segments up to day 21 after pacing. Segmental shortening, as measured by sonomicrometry, also was significantly decreased at day 7 in the AdNull group compared with that in the $\mathrm{Ad}_{\mathrm{CU}}$ VEGF121.1 group $(10 \% \pm 4 \%$ vs $16 \% \pm 3 \%, P=$ $.004)$ and remained significantly impaired $(P<.05)$ in the AdNull group at day 14 and 21 when compared with baseline values. 


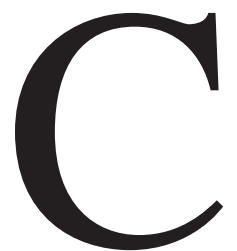

oronary artery disease remains the leading cause of death in the United States today. ${ }^{1}$ In patients with coronary disease, the leading cause of death is congestive heart failure. ${ }^{2,3}$ In turn, the leading cause of congestive heart failure is myocardial ischemia.

Heart failure caused by myocardial ischemia is typically associated with the occurrence of hibernating myocardium, viable tissue that is nevertheless poorly functioning or noncontractile because of severe ischemia. ${ }^{4-6}$ It is thought that this phenomenon serves as a protective mechanism, downregulating energy consumption to preserve cell viability. By definition, however, myocardial hibernation and restoration of myocardial function is reversible with myocardial reperfusion.

Although myocardial ischemia caused by discrete coronary stenosis can usually be treated with coronary angioplasty or bypass graft surgery, diffuse coronary artery disease is commonly not amenable to these conventional therapies. On the basis of these considerations, we hypothesized that improvement in blood flow to the myocardium induced by treatment with angiogenic factors could be used to improve myocardial dysfunction in the setting of ischemic cardiomyopathy.

Vascular endothelial growth factor (VEGF) is a homodimeric 34- to 44-kd heparin-binding glycoprotein that is a potent angiogenic growth factor (angiogen). As a result of posttranscriptional mRNA splicing, VEGF normally exists as 4 major isoforms of 121-, 165-, 189-, and 206-amino-acid residues. ${ }^{7}$ VEGF is an attractive candidate for therapeutic angiogenic applications in that, because of localization of its receptors almost exclusively to vascular endothelial cells, it is one of the most specific of the known angiogens.

We and others have used gene transfer as a delivery strategy in which the coding sequence for a selected angiogen, such as VEGF, is delivered to the target tissue to induce angiogenesis..$^{8-12}$ Although encouraging initial data have demonstrated improvement in regional myocardial function in the setting of discrete coronary obstructions with this therapy, ${ }^{8-10}$ the efficacy of angiogenic therapy in reversing global myocardial dysfunction is largely unknown.

In the present study we compared the efficacy of intramyocardial administration of an adenovirus coding for the 121-amino-acid isoform of VEGF $\left(\mathrm{Ad}_{\mathrm{CU}}\right.$ VEGF121.1) with that of a vector coding for no genes (AdNull) in enhancing myocardial function in a porcine pacing model of cardiomyopathy. Data generated in this study suggest that $\mathrm{Ad}_{\mathrm{CU}}$ VEGF121.1 administration helps to preserve and enhance recovery of cardiac function in this model of ischemic cardiomyopathy. This may represent a novel strategy for treating cardiomyopathy in the ischemic setting.

\section{Methods}

\section{Experimental Model for Heart Failure}

A model of cardiomyopathy was created in male Yorkshire swine (25-30 kg) by using rapid ventricular pacing. ${ }^{13,14}$ Care provided for animals was in compliance with the US National Research Council's "Guide for the Care and Use of Laboratory Animals" and proceeded under protocols approval by the Evanston Northwestern Healthcare Institutional Animal Care Committee. Initial anesthesia was performed with telazol $(4 \mathrm{mg} / \mathrm{kg})$ and xylazine (2 $\mathrm{mg} / \mathrm{kg}$ ) and maintained with isoflurane (0.5\%-2.0\%) after intubation. A posterolateral thoracotomy was performed, and 2-mm piezoelectric crystals (Sonometrics, London, Ontario, Canada) were implanted in the myocardium at the apex and at the base of the left ventricle under the left atrial appendage (for long-axis measurements) and anteriorly and posteriorly at the midpapillary level (for short-axis measurements).

After initial testing of data acquisition and analysis with the Digital Ultrasonic Measurement System and the Sonosoft software program 3.1 (Sonometrics), the sonomicrometry leads were exteriorized for later use. An epicardial pacing lead (Medtronic, Minneapolis, Minn) was placed at the left ventricular apex and attached to a single-chamber pacing generator (Medtronic), which was placed subcutaneously. After a 7-day recovery period, pacing at 230 beats/min was initiated and continued for 7 days. Pacing was well tolerated in all animals. (There were 2 deaths each in the AdNull and the $\mathrm{Ad}_{\mathrm{CU}}$ VEGF121.1 groups: one before and one immediately after pacing in the $\mathrm{Ad}_{\mathrm{CU}} \mathrm{VEGF} 121.1$ group and both immediately after pacing in the AdNull group.)

Echocardiographic and sonomicrometric data were collected at the time of the operation, immediately before pacing (baseline; 7 days postoperatively), immediately after pacing (day $0 ; 14$ days postoperatively), and weekly thereafter for 3 weeks before the animals were killed (days 7, 14, and 21). All echocardiographic and sonomicrometric data were recorded for subsequent analysis, except for some instances in which results were uninterpretable or unavailable because of poor image quality or lead displacement, respectively. 


\section{Adenovirus Vectors}

The replication-deficient vector $\mathrm{Ad}_{\mathrm{CU}}$ VEGF121.1 is an E1a ${ }^{-}$, partial E1b ${ }^{-}$, partial E3 ${ }^{-}$adenovirus vector based on human adenovirus 5 into which an expression cassette is inserted into the E1 region containing the cytomegalovirus immediate early promoterenhancer, an artificial splice sequence, the human VEGF 121 cDNA, and the SV40 polyA/stop signal. ${ }^{15}$ The vector AdNull (containing no transgene) served as the control. $\mathrm{Ad}_{\mathrm{CU}}$ VEGF121.1induced VEGF expression was confirmed by means of enzymelinked immunosorbent assay (R\&D Systems Inc, Minneapolis, Minn) assessment of tissue-culture supernatant 48 hours after in vitro infection of A549 (American Type Culture Collection, Rockville, Md).

\section{Intramyocardial Vector Delivery}

In initial experiments naive animals undergoing crystal placement and pacing, as described above, but without vector injection, were analyzed to assess the specific effects of pacing alone on ventricular function $(n=5)$. Subsequently, at the time of the initial operation, animals were administered either $\mathrm{Ad}_{\mathrm{CU}}$ VEGF121.1 $(\mathrm{n}=8)$ or the control vector AdNull $(\mathrm{n}=8)$ by means of direct myocardial injection of the vector in phosphate-buffered saline solution at 10 sites $(10 \mu \mathrm{L} / \mathrm{site})$ placed at $1-\mathrm{cm}$ intervals in the anterior and lateral territory of the left ventricle (total dose, $10^{11}$ $\mathrm{pu})$.

\section{Echocardiographic Assessment}

Two-dimensional transthoracic images were obtained with an Acuson echocardiographic machine (128 XP/10; Acuson, Mountain View, Calif) and a 3.0/3.5-MHz dual-frequency transducer (Acuson S 3194). During each examination, animals were sedated and placed in the left lateral decubitus position, and right parasternal short-axis midpapillary views of the left ventricle were obtained at rest. Images were recorded for 3 minutes on a standard VHS videotape. Offline analysis of the data was performed by a single experienced investigator, who was blinded to group assignment, using the Image Vue DCR 1.50 System (Nova Microsonics, Mahwah, NJ).

Global ventricular function was estimated by calculating the fractional area change (FAC) of the left ventricle. The percentage of FAC has been shown to correlate well with radionuclide and angiography measurements of ejection fraction. ${ }^{16}$ The percentage of FAC was measured by tracing the end-diastolic area (EDA) and the end-systolic area (ESA) of the ventricle from the midpapillary short-axis view. The percentage of FAC was then calculated by using the following equation:

$$
\% \mathrm{FAC}=[(\mathrm{EDA}-\mathrm{ESA}) / \mathrm{EDA}] \times 100 .
$$

Regional wall-motion function was evaluated by dividing the left ventricle into 6 equal $60^{\circ}$ radial segments (Figure 1). Mean systolic and mean diastolic wall thickness were calculated by averaging 3 single measurements in each segment.

Systolic wall thickening in each segment was defined as the mean systolic wall thickness minus the mean diastolic wall thickness. Fractional wall thickening (FWT) was defined as the mean systolic wall thickening times 100 divided by the mean diastolic wall thickness.

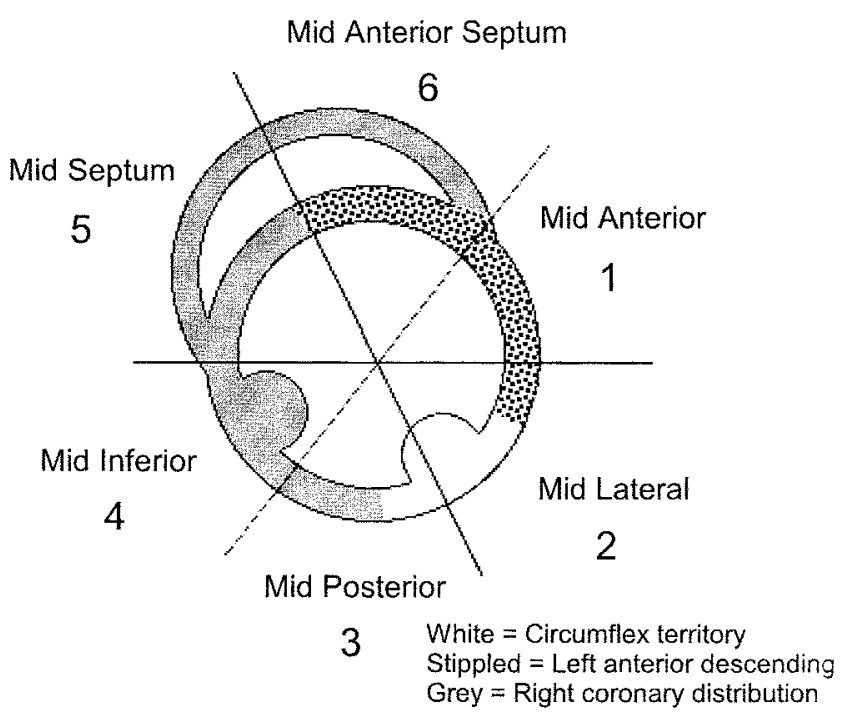

Figure 1. Schematic of ventricular sectioning in the short axis at the midventricular (midpapillary) level for echocardiographic analysis of FWT.

\section{Sonomicrometric Assessment}

Heart rate, segmental shortening, stroke volume, and cardiac output were measured with a commercial digital sonomicrometry system (Sonometrics Corp, London), according to the manufacturer's specifications, before (rest) and after (stress) administration of epinephrine (10 $\mu \mathrm{g}$ administered intravenously) at each hemodynamic measurement time point. ${ }^{17}$ The scalar distances between omnidirectional sonomicrometers (pairs of 1.3-MHz piezoelectric crystals) were recorded simultaneously at a sampling frequency of greater than $240 \mathrm{~Hz}$. Parameters derived from the intercrystal distances and heart rate were calculated by using a 4-component system consisting of (1) a 486-class personal computer that controls sonomicrometry functions and runs application software, (2) a customized timing board that controls the length of the energizing pulse and the sequence of transducer transmissions, (3) a customized distance-measuring circuit that translates the ultrasonic signal into a distance measurement, and (4) an 8-channel analogto-digital board that is synchronized with the sonomicrometer distance measurements.

\section{Statistical Analysis}

Data are reported as means and SDs, and significance measurements were calculated with paired or unpaired Student $t$ tests, as appropriate.

\section{Results}

\section{Pacing Model}

In initial experiments conducted in naive animals without vector administration, pacing results in a decrease in ventricular function that persisted up to 21 days after cessation of pacing. Segmental shortening, for example, decreased from $13.5 \% \pm 4.6 \%$ before pacing to $5.6 \% \pm 3.0 \%$ at day 0 after pacing and $6.8 \% \pm 3.0 \%$ at day 7 after pacing $(P<$ 


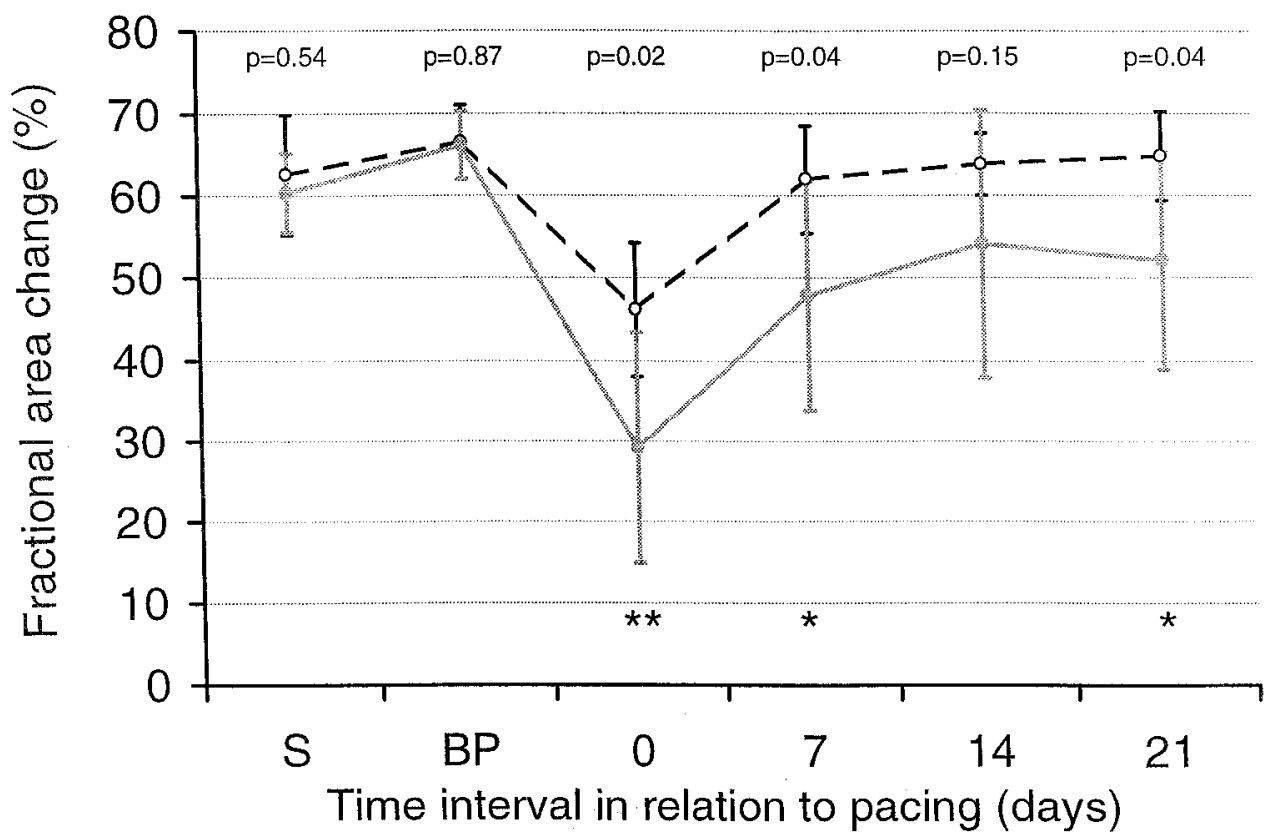

Figure 2. Echocardiographic assessment of the percentage of FAC of the left ventricular cavity (systole vs diastole) in the short axis at the midventricular (midpapillary) level calculated as described in the "Methods" section (mean \pm SD). $S$, Initial operation; $B P$, before pacing; $0,7,14$, and 21, days after cessation of pacing. Statistical comparisons between $\mathrm{Ad}_{\mathrm{cu}}$ VEGF121.1 and AdNull groups are provided above the corresponding time point. Statistically significant comparisons for AdNull at various time points and at baseline (BP) are provided as indicated $\left(* P<.05,{ }^{* *} P<.01\right)$.

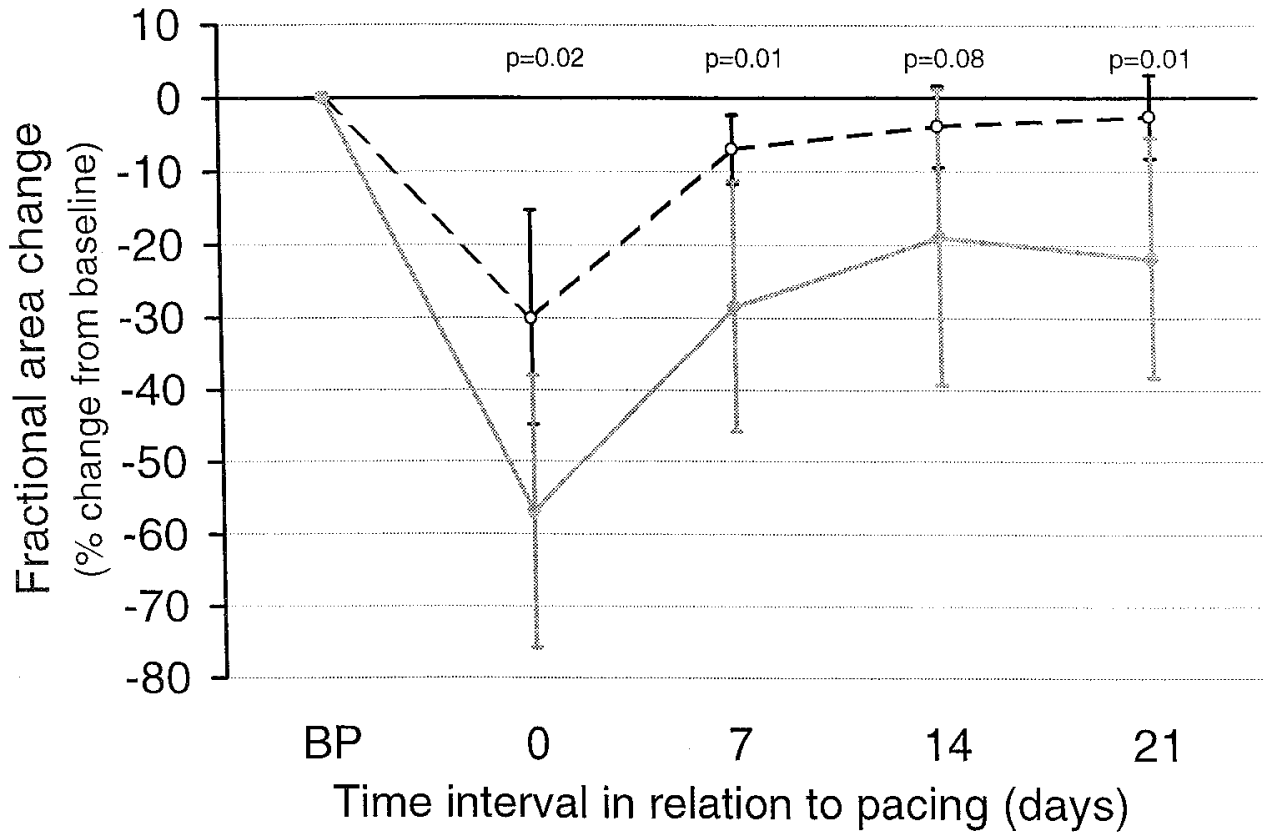

Figure 3. Echocardiographic assessment of the percentage of FAC of the left ventricular cavity (systole vs diastole) in the short axis at the midventricular (midpapillary) level calculated as the percentage of change (mean \pm SD) of each animal from its corresponding prepacing baseline value, as described in the "Methods" section. BP, Before pacing; 0, 7, 14, and 21, days after cessation of pacing. Statistical comparisons between the $\mathrm{Ad}_{\mathrm{cu}} \mathrm{VEGF121.1}_{\text {and }}$ AdNull groups are provided above the corresponding time point. Statistically significant comparisons for AdNull at various time points and at baseline $(B P)$ are provided as indicated $\left({ }^{*} P<.05,{ }^{*} P<.01\right)$. 


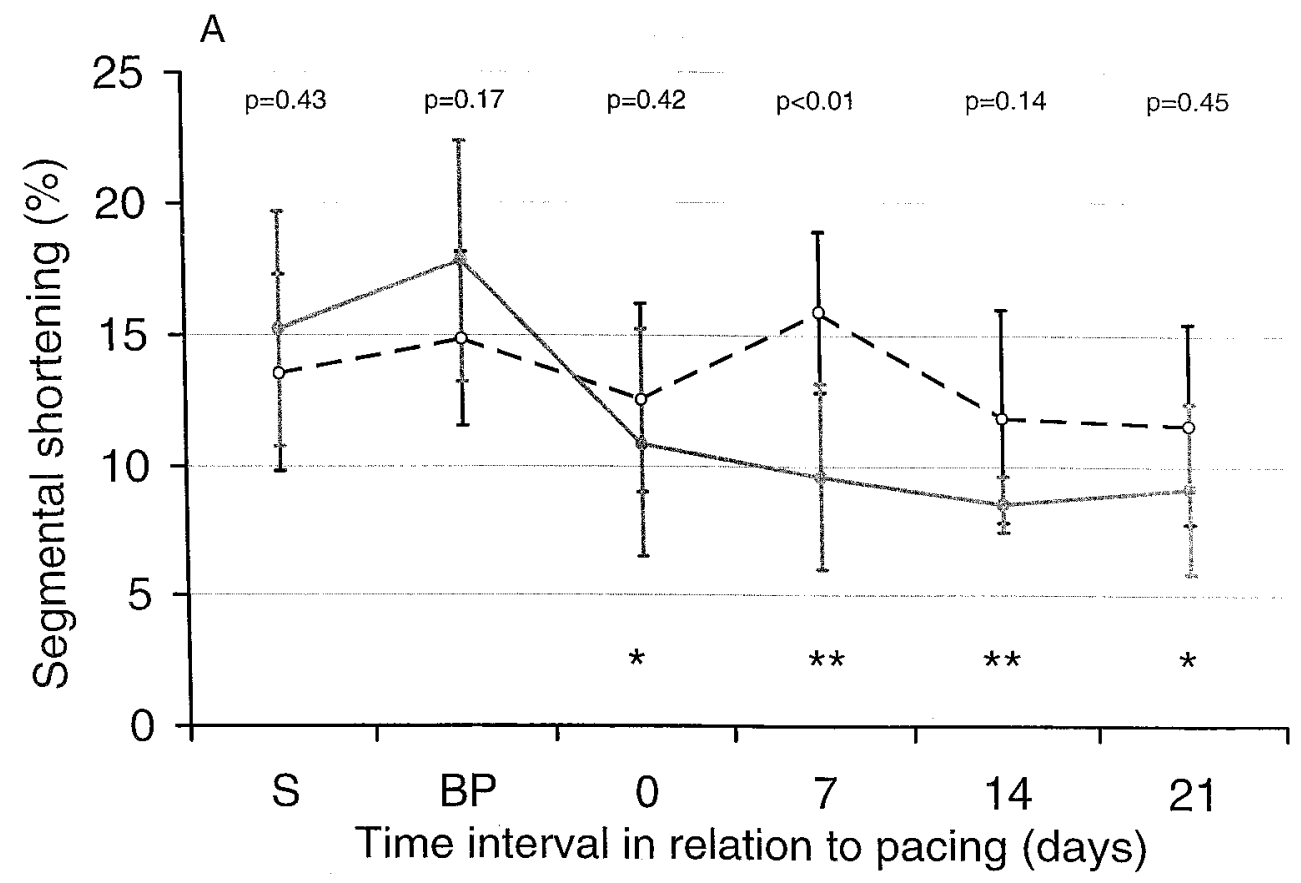

B

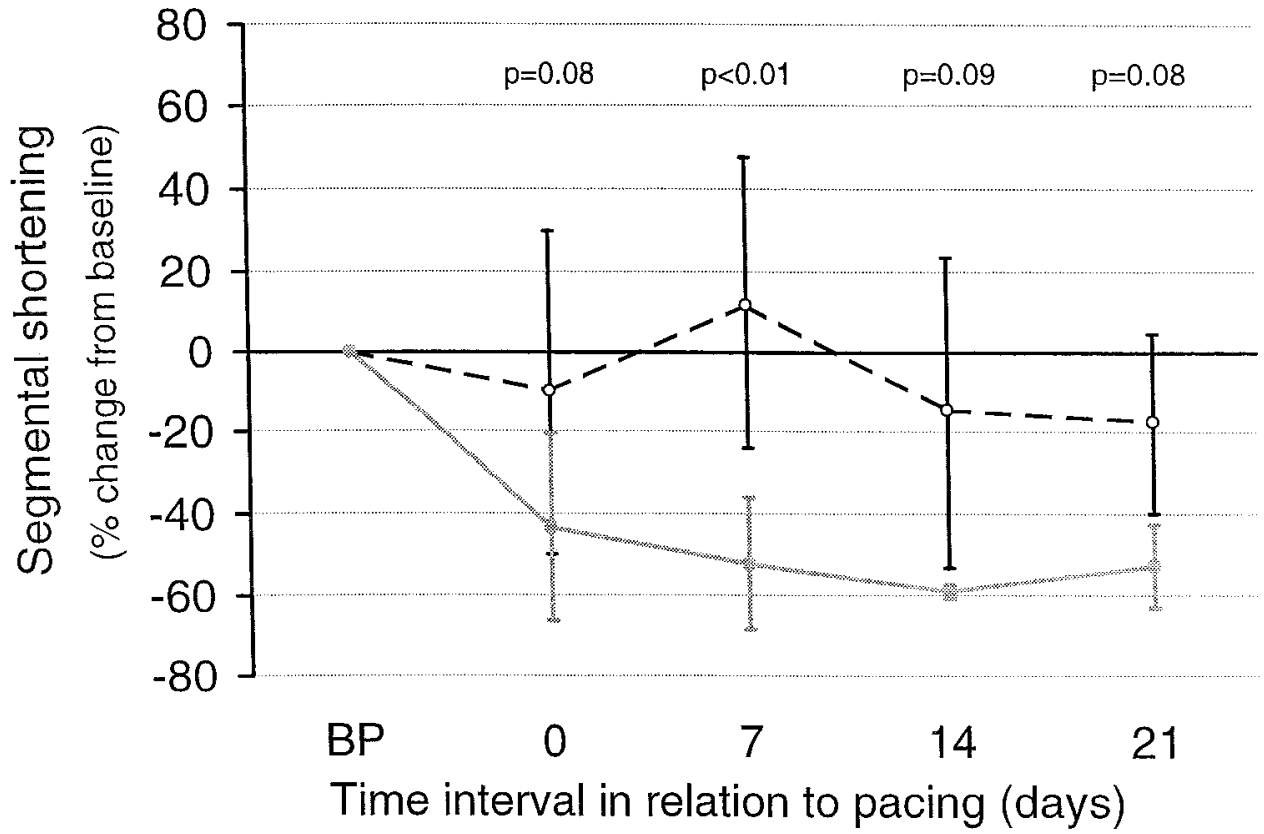

Figure 4. Sonomicrometric assessment of ventricular function. All analyses are based on measurements with piezoelectric crystals of short- and long-axis dimensions in systole versus diastole calculated as described in the "Methods" section. Depicted are absolute values and percentage changes from the prepacing baseline value of each animal (mean $\pm S D$ ), respectively, in short-axis segmental shortening (A and $B$ ), stroke volume (C and D), and cardiac output (E and $F$ ). $S$, Initial operation; $B P$, before pacing; $0,7,14$, and 21, days after cessation of pacing. Values are $\mathrm{Ad}_{\mathrm{cu}}$ VEGF121.1 (open circles) versus AdNull (filled circles). Statistical comparisons between $\mathrm{Ad}_{\mathrm{cu}}$ VEGF121.1 and AdNull groups are provided above the corresponding time point. Statistically significant comparisons for AdNull at various time points and at baseline (BP) are provided as indicated $\left.{ }^{*} \boldsymbol{P}<.05,{ }^{*} P<.01\right)$. 

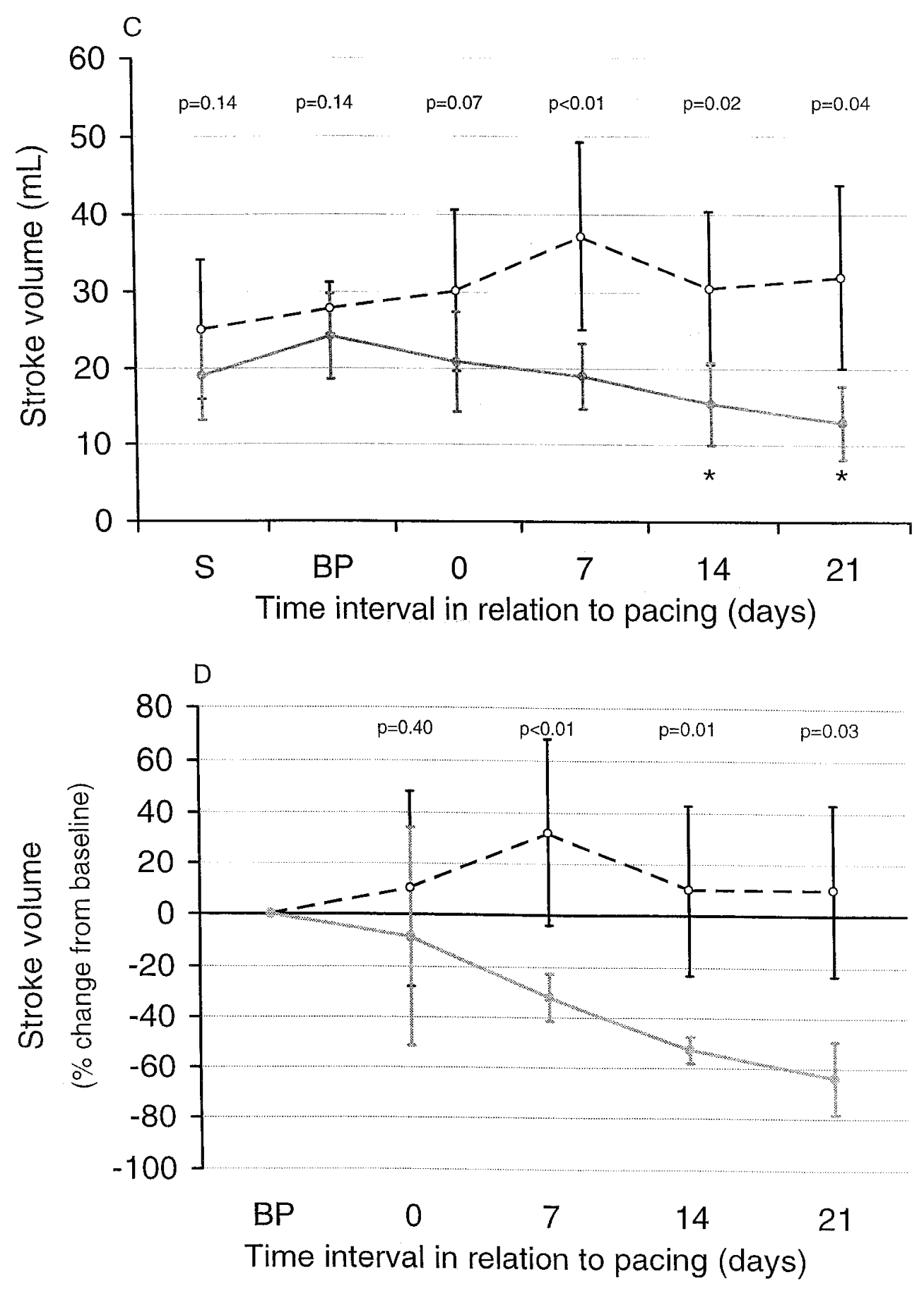

Figure 4. Cont'd.

.03 ) and remained decreased at $9.8 \% \pm 5.8 \%$ up to 21 days after pacing.

Echocardiographic assessment of ventricular morphology in the treated animals at day 21 after pacing also demonstrated evidence consistent with the presence of congestive failure. For example, end-diastolic area in the $\mathrm{Ad}_{\mathrm{CU}}$ VEGF121.1 animals was $910 \pm 200 \mathrm{~mm}^{2}$ versus
$1120 \pm 230 \mathrm{~mm}^{2}$ in the AdNull group. End-systolic areas were $350 \pm 110 \mathrm{~mm}^{2}$ and $560 \pm 180 \mathrm{~mm}^{2}$, respectively.

\section{Echocardiographic Assessment of Global Ventricular} Function

Echocardiographic assessment of FAC at the midventricular level did not differ between the $\mathrm{Ad}_{\mathrm{CU}}$ VEGF121.1 and 

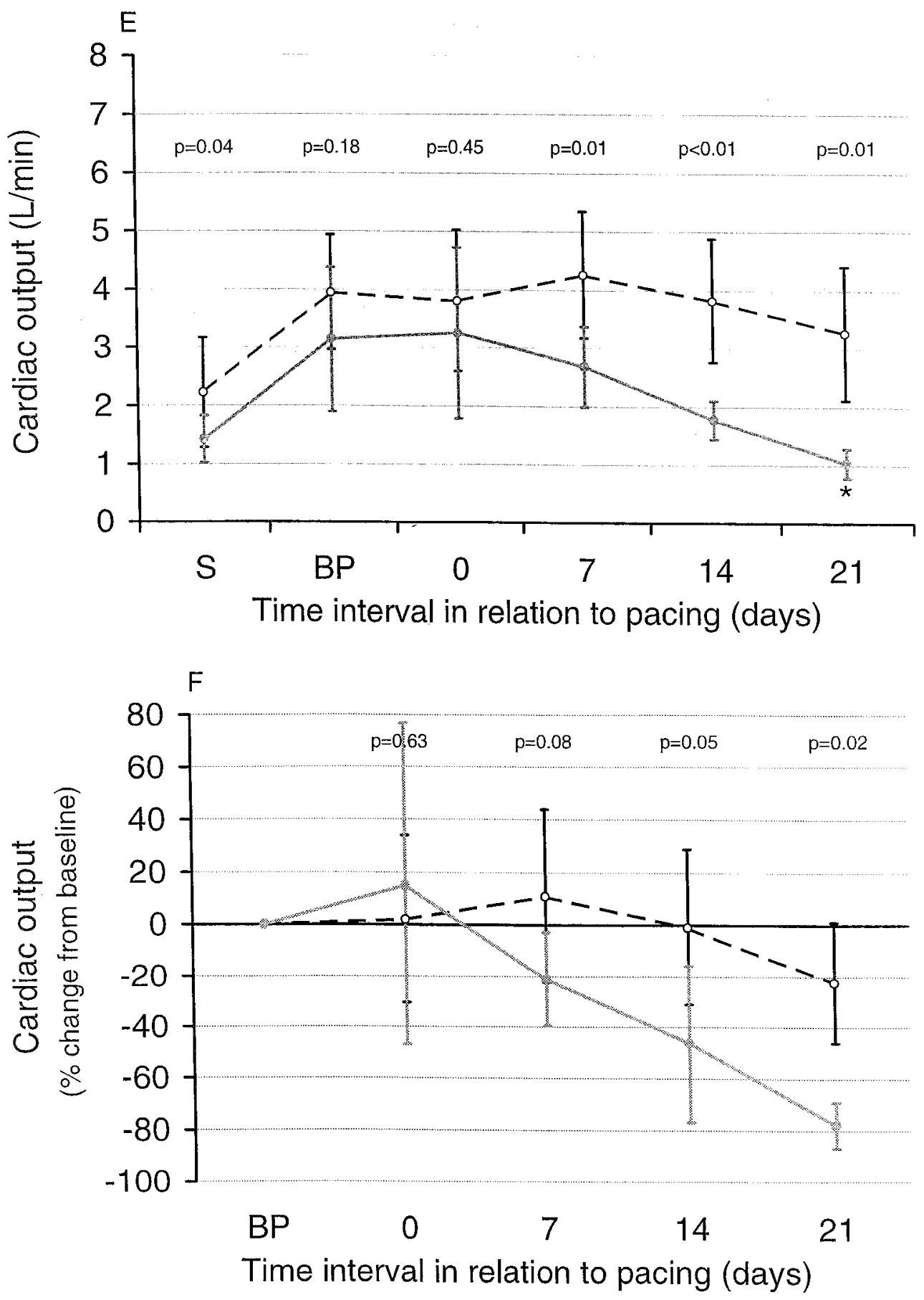

Figure 4. Cont'd.

AdNull groups before initiation of pacing $(67 \% \pm 5 \%$ vs $66 \% \pm 4 \%, P=.9$; Figure 2). FAC significantly decreased on termination of pacing in both groups but was significantly greater in the $\mathrm{Ad}_{\mathrm{CU}}$ VEGF121.1 group compared with that in the AdNull group at this time point $(46 \% \pm 8 \%$ vs $29 \% \pm 14 \%, P=.02$ ). Ventricular function improved in both groups over succeeding time points, but although the
FAC in the $\mathrm{Ad}_{\mathrm{CU}} \mathrm{VEGF121.1}$ group recovered to levels approximating prepacing values by day 7 after pacing, FAC in the AdNull group remained significantly decreased compared with $\mathrm{Ad}_{\mathrm{CU}}$ VEGF121.1 levels throughout the 3-week period (day 7: $48 \% \pm 14 \%$ vs $62 \% \pm 7 \%, P<.04$; day 21 : $52 \% \pm 13 \%$ vs $65 \% \pm 5 \%, P<.04)$.

The differences between the $\operatorname{Ad}_{\mathrm{CU}} \mathrm{VEGF121.1}$ and 
A

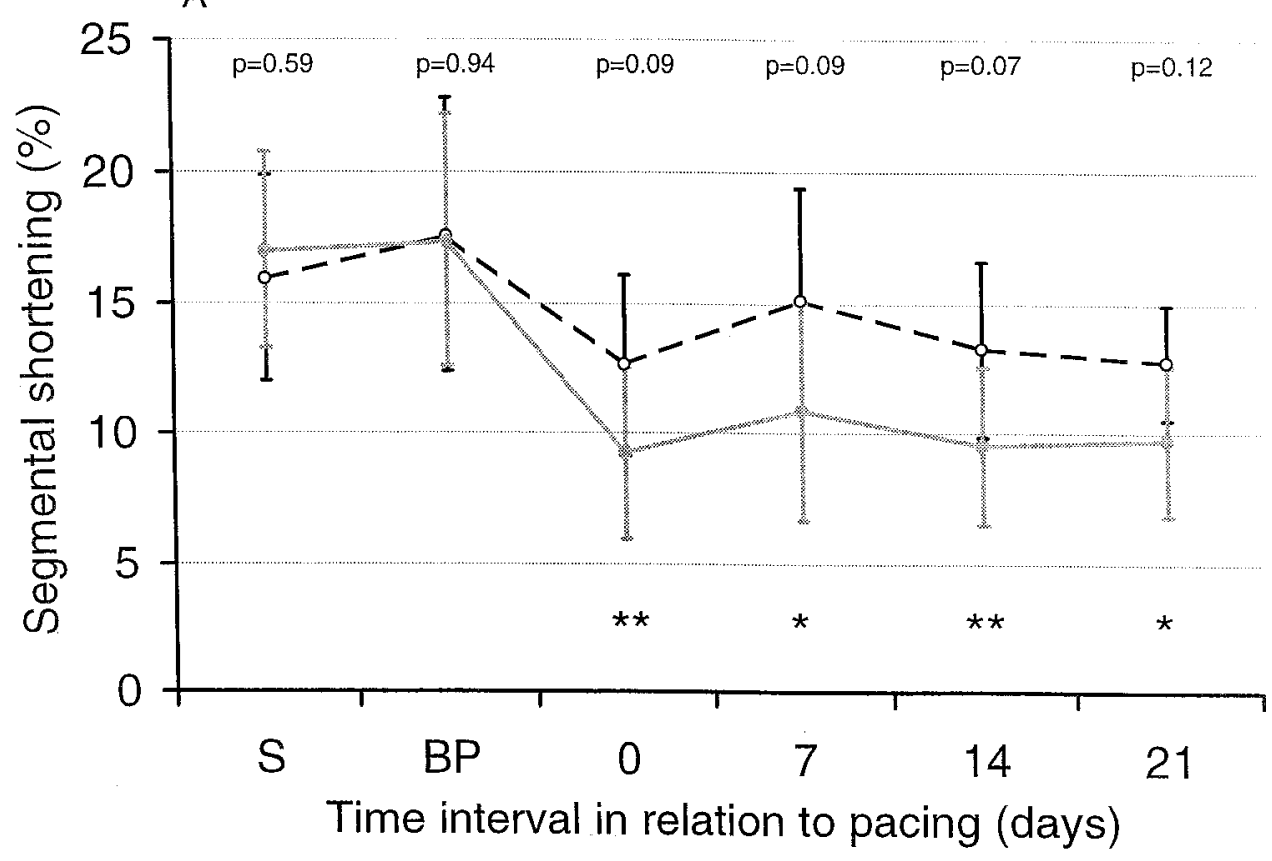

B

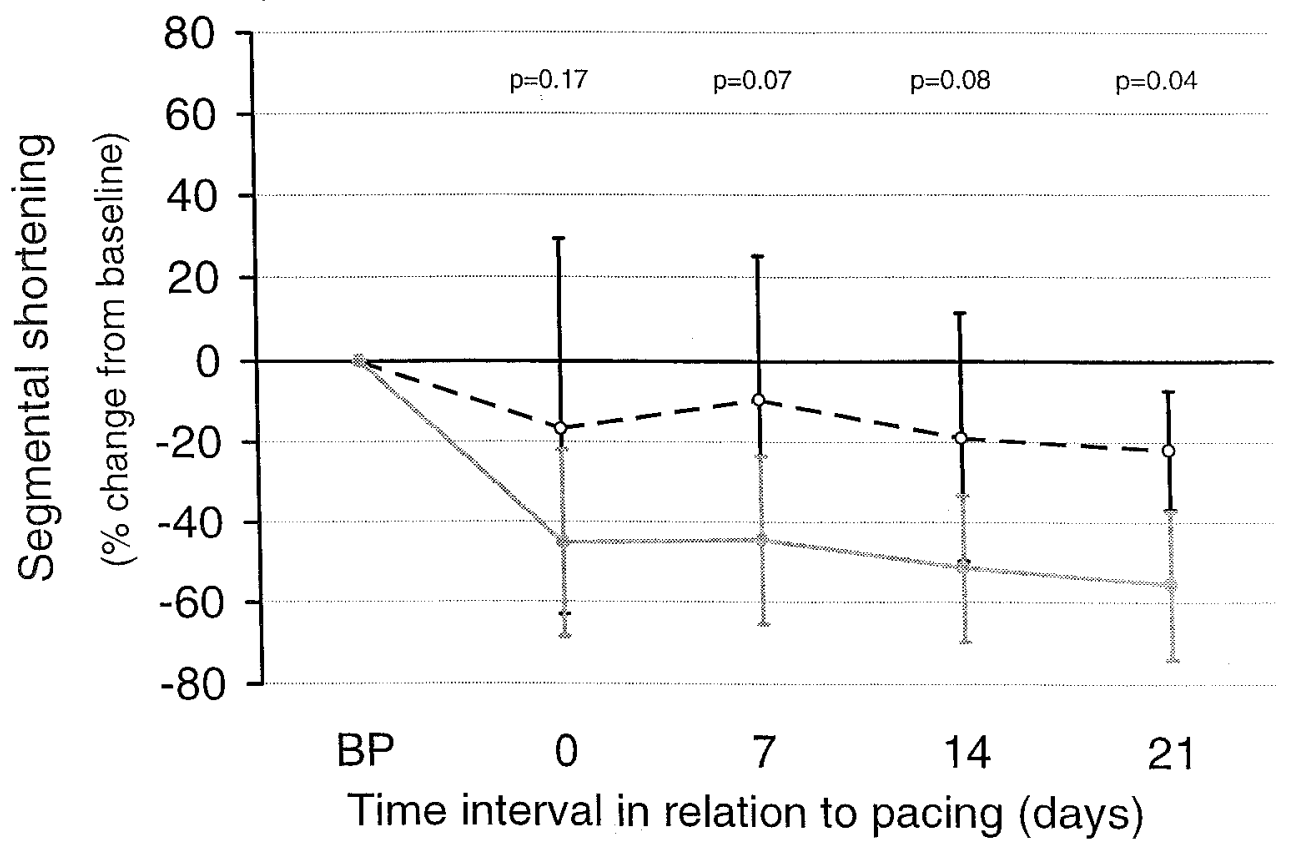

Figure 5. Sonomicrometry assessment of ventricular function after administration of epinephrine. All analyses are based on measurements from piezoelectric crystals measuring short- and long-axis dimensions in systole versus diastole calculated as described in the "Methods" section. Depicted are absolute values and percentage changes from the prepacing baseline value of each animal (mean $\pm S D)$, respectively, in short-axis segmental shortening ( $A$ and $B$ ), stroke volume (C and D), and cardiac output (E and $F$ ). $S$, Initial operation; $B P$, before pacing; $0,7,14$,

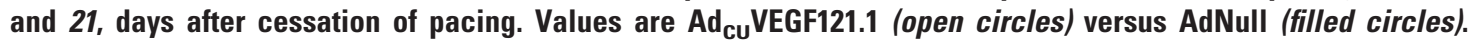
Statistical comparisons between $\mathrm{Ad}_{\mathrm{cu}}$ VEGF121.1 and AdNull groups are provided above the corresponding time point. Statistically significant comparisons for AdNull at various time points and at baseline (BP) are provided as indicated $\left(* P<.05,{ }^{* *} P<.01\right)$. 

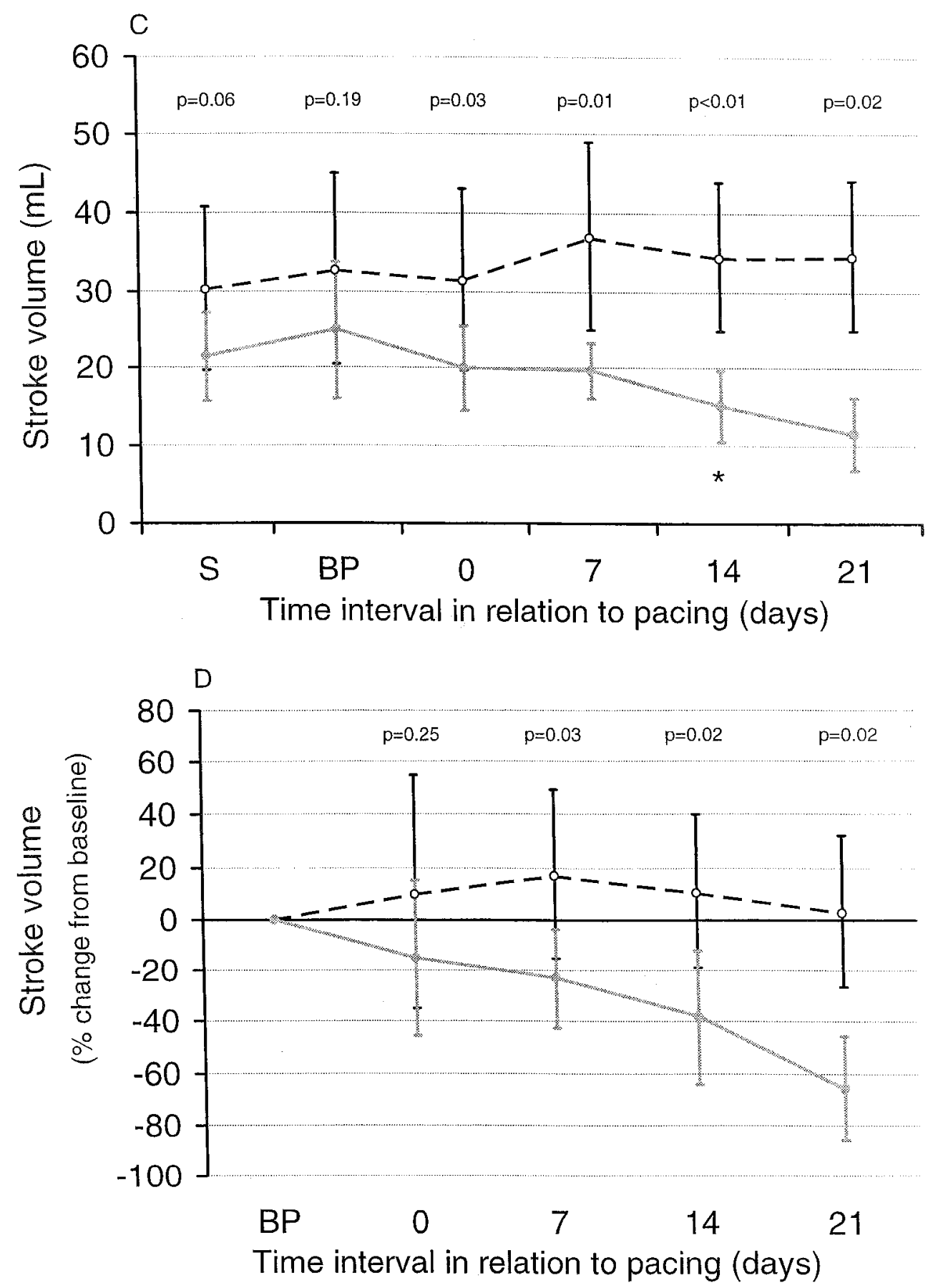

Figure 5. Cont'd.

AdNull groups were more pronounced if changes in FAC were plotted as a function of change from each animal's own prepacing baseline measurements (Figure 3). For example, by using this analysis, the $\mathrm{Ad}_{\mathrm{CU}}$ VEGF121.1 animals demonstrated a decrease in FAC of no more than about $7 \%$ at any time point at or beyond 7 days after pacing compared with prepacing values, whereas AdNull animals demon- strated a decrease from baseline in FAC that was $22 \% \pm$ $16 \%$ at 21 days after pacing $(P<.02)$.

\section{Echocardiographic Assessment of FWT}

FWT was similar in all 6 midventricular segments in both the $\mathrm{Ad}_{\mathrm{CU}}$ VEGF121.1 and AdNull groups before pacing $\left(\mathrm{Ad}_{\mathrm{CU}}\right.$ VEGF121.1 vs AdNull: $37 \% \pm 6 \%$ vs $39 \% \pm 5 \%$ 

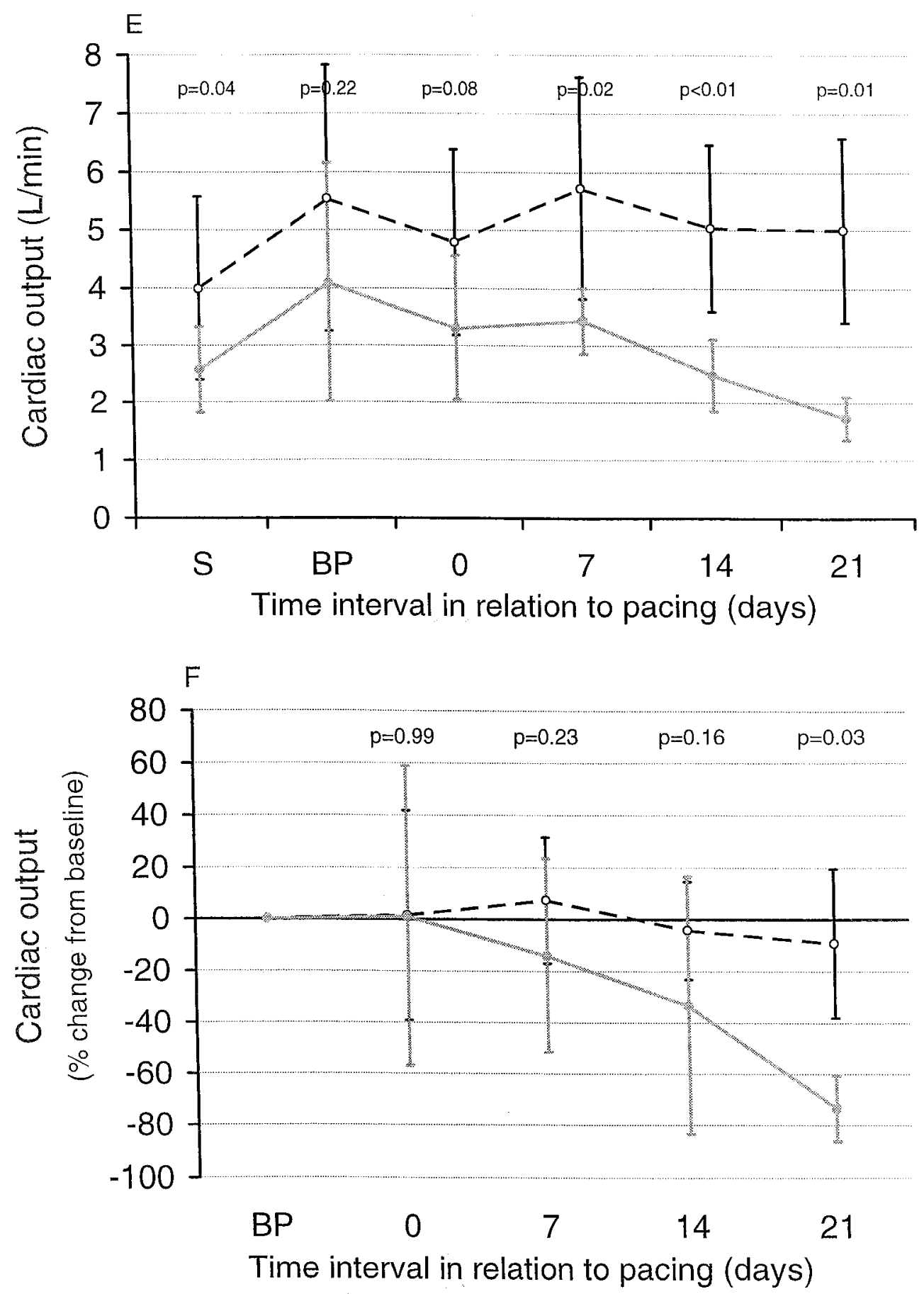

Figure 5. Cont'd.

[mean of all segments]) but demonstrated a decrease at day 0 after pacing that was greater $(P<.05)$ in the AdNull group compared with that in the $\mathrm{Ad}_{\mathrm{CU}}$ VEGF121.1 group in 5 of 6 segments. The average FWT for all segments improved to $36 \% \pm 5 \%$ within 7 days after pacing in the $\mathrm{Ad}_{\mathrm{CU}}$ VEGF121.1 group, approximating prepacing levels, but remained significantly impaired $(P \leq .04)$ compared with prepacing FWT in the AdNull group in 5 of 6 segments, even at day 21.

\section{Sonomicrometric Assessment of Hemodynamic}

Performance

The prepacing values for segmental shortening did not differ significantly between the $\mathrm{Ad}_{\mathrm{CU}}$ VEGF121.1 and the 
TABLE 1. Cardiac output (L/min)

\begin{tabular}{|c|c|c|c|c|}
\hline & \multicolumn{2}{|c|}{ Rest } & \multicolumn{2}{|c|}{ Stress } \\
\hline & AdNull & $\mathrm{Ad}_{\mathrm{Cu}}$ VEGF121.1 & AdNull & $\mathrm{Ad}_{\mathrm{Cu}}$ VEGF121.1 \\
\hline Initial operation & $1.4 \pm 0.4$ & $2.2 \pm 0.9$ & $2.6 \pm 0.8$ & $4.0 \pm 1.6$ \\
\hline Before pacing* & $3.1 \pm 1.2$ & $4.0 \pm 1.0$ & $4.1 \pm 2.1$ & $5.5 \pm 2.3$ \\
\hline \multicolumn{5}{|l|}{ Days after pacing } \\
\hline 0 & $3.3 \pm 1.5$ & $3.8 \pm 1.2$ & $3.3 \pm 1.3$ & $4.8 \pm 1.6$ \\
\hline 7 & $2.7 \pm 0.7$ & $4.3 \pm 1.1$ & $3.4 \pm 0.6$ & $5.7 \pm 1.9$ \\
\hline 14 & $1.8 \pm 0.3$ & $3.8 \pm 1.1$ & $2.5 \pm 0.6$ & $5.0 \pm 1.4$ \\
\hline 21 & $1.0 \pm 0.3 \dagger$ & $3.3 \pm 1.1$ & $1.7 \pm 0.4$ & $5.0 \pm 1.6$ \\
\hline
\end{tabular}

* Seven-day recovery period provided after the initial operation.

$\dagger P=.02$ compared with baseline values.

AdNull groups (Figure 4, A). Segmental shortening was not significantly impaired in the $\mathrm{Ad}_{\mathrm{CU}}$ VEGF121.1 group at any time after pacing compared with the prepacing values. In contrast, segmental shortening at day 7 was significantly decreased in the AdNull group compared with that in the $\mathrm{Ad}_{\mathrm{CU}}$ VEGF121.1 group $(10 \% \pm 4 \%$ vs $16 \% \pm 3 \%, P=$ $.004)$ and remained significantly impaired $(P<.05)$ in the AdNull group at day 14 and 21 when compared with baseline values. These differences were more dramatically demonstrated when the postpacing function of each animal was expressed as a percentage change from its own baseline (immediate prepacing) values (Figure 4, $B$ ).

Stroke volume was also equivalent in the $\mathrm{Ad}_{\mathrm{CU}}$ VEGF121.1 group compared with that in the AdNull animals before pacing and did not significantly decrease from these baseline values in the $\mathrm{Ad}_{\mathrm{CU}}$ VEGF121.1 group at any time during the postpacing study period (Figure $4, C$ ). In contrast, stroke volume in the AdNull group became significantly more impaired throughout the postpacing period (day 21: $P<.02$ ). Depression of stroke volume was most pronounced compared with corresponding values for the $\mathrm{Ad}_{\mathrm{CU}}$ VEGF121.1 group at day 7 after pacing (19 \pm 4 vs $37 \pm 12 \mathrm{~mL}, P=.004$ ) but was also impaired as compared with that of the $\mathrm{Ad}_{\mathrm{CU}}$ VEGF121.1 animals at day 14 (15 \pm 5 vs $31 \pm 10 \mathrm{~mL}, P<.02)$ and at day $21(13 \pm 5$ vs $32 \pm$ $12 \mathrm{~mL}, P<.04)$. This impairment in ventricular function in AdNull animals was also demonstrated when comparing the postpacing results of each animal with its corresponding baseline value (Figure 4, D).

Cardiac outputs were similar for the 2 groups immediately before pacing, although they were greater for the $\mathrm{Ad}_{\mathrm{CU}}$ VEGF121.1 animals compared with those seen in the AdNull animals at the time of the initial operation (Figure 4, $E$ ). Cardiac outputs were well preserved in the $\mathrm{Ad}_{\mathrm{CU}}$ VEGF121.1 group compared with those in the AdNull group throughout the study (day $7: 4.3 \pm 1.1$ vs $2.7 \pm 0.7$ $\mathrm{L} / \mathrm{min}, P=.009$; day $14: 3.8 \pm 1.1$ vs $1.8 \pm 0.3 \mathrm{~L} / \mathrm{min}, P=$ .004 ; day $21: 3.3 \pm 1.1$ vs $1.0 \pm 0.3 \mathrm{~L} / \mathrm{min}, P<.02)$. Even at day 21 after pacing, cardiac output in the AdNull group remained significantly impaired $(P<.03)$ when compared with each animal's own baseline values (Figure $4, F$ ).

\section{Assessment of Ventricular Performance With Pharmacologic Stress}

Sonomicrometry was used to assess the effect of epinephrine administration ( $10 \mu \mathrm{g}$ administered intravenously) on cardiac performance (Figure 5, A-F). Before pacing, epinephrine administration resulted in an approximate $30 \%$ to $85 \%$ increase in cardiac contractility, as measured by changes in cardiac output (Table 1). Administration of epinephrine failed to restore any of the measured indices of ventricular performance in AdNull animals to levels equivalent to those demonstrated by $\mathrm{Ad}_{\mathrm{CU}}$ VEGF121.1 animals in the resting state. Similarly, epinephrine administration resulted in a mean increase in cardiac output in $\mathrm{Ad}_{\mathrm{CU}}$ VEGF121.1 animals that was nearly double that of the increase in cardiac output noted after epinephrine administration in AdNull animals (Table 1).

\section{Discussion}

Despite many recent advances in the treatment of coronary artery disease, including the application of angioplasty and coronary bypass surgery to treat discrete lesions in the large epicardial vessels, treatment options for patients with endstage ischemic cardiomyopathy remain limited. Such patients characteristically have diffuse coronary disease that is often not amenable to treatment with conventional methodologies. Alternative treatment strategies, such as cardiac transplantation, implantation of an artificial assist device, or multidrug pharmacotherapy remain limited by significant associated morbidities and toxicities. Thus there remains a significant need for effective alternative therapies for cardiomyopathy.

Angiogenic therapy appears to offer promise as an effective means of enhancing the function of ischemic myocardium by improving blood flow to ischemic tissues through increased myocardial vascularization. ${ }^{8-12}$ In contrast, the treatment of heart failure with chronic inotropic 
therapy has been associated with excessive mortality, possibly because this strategy may exacerbate preexistent ischemia. ${ }^{18}$

Evidence supporting the ability of growth factor administration to induce therapeutic angiogenesis has been provided by a wide variety of animal studies and a limited number of early clinical trials. ${ }^{8-12,19-30}$ Gene therapy, whereby the angiogenic agent is transferred to the myocardium by means of a viral or plasmid vector as the cDNA for the corresponding protein angiogen, is a promising delivery strategy for angiogenic therapy. ${ }^{19}$ In the present study we demonstrate that adenoviral-mediated transfer of VEGF 121 as an angiogenic mediator appears to minimize the development of, and speed recovery from, ventricular dysfunction induced by rapid ventricular pacing. The findings of the present study suggest that this intervention may represent an effective treatment for cardiomyopathy.

Enhancement of myocardial performance after delivery of the $\mathrm{Ad}_{\mathrm{CU}}$ VEGF121.1 vector was demonstrated by means of both echocardiographic and sonomicrometric assessment of ventricular function, by using both global and regional analyses. In comparison with prior studies demonstrating that angiogenic therapy improves regional myocardial perfusion and function, ${ }^{8-10,20-22}$ angiogenic therapy in the present study was used to treat global dysfunction and is thus more relevant for the treatment of ischemic cardiomyopathy. In contrast to the 2 previous studies assessing the treatment of global dysfunction with intracoronary delivery of a growth factor, ${ }^{11,31}$ the present study demonstrates the efficacy of an intramyocardial delivery technique. This approach takes advantage of the potentially beneficial localizing effects of intramyocardial delivery ${ }^{9,32}$ and suggests that such a strategy is a viable means of homogeneously enhancing global function, despite the use of multiple, isolated injections.

Several limitations of this study must be addressed. First, we did not specifically document the induction of angiogenesis in the current study, although clear evidence of enhanced vascularization, perfusion, function, or a combination thereof has been previously established after myocardial delivery of angiogenic therapy, ${ }^{8-12,24,26,27,33,34}$ including our recent demonstration of enhanced myocardial perfusion after $\mathrm{Ad}_{\mathrm{CU}} \mathrm{VEGF121.1}$ delivery in the nonischemic rabbit myocardium (G. F. Rahman, unpublished observations). Although it is possible that nonangiogenic effects of VEGF, such as vasodilator or cytoprotective properties, ${ }^{12}$ may have played a role in the hemodynamic changes noted in this study, the pharmacokinetics of adenovirus delivery, whereby transgene expression beyond 2 weeks is negligible, ${ }^{9,32}$ suggest that this alternative mechanism is unlikely.

A second limitation is that the rapid ventricular pacing model we used does not duplicate the diffuse coronary occlusions and multiple myocardial infarctions typically associated with ischemic cardiomyopathy in the clinical setting. Myocardial ischemia in our model is likely induced by excessive myocardial demand induced by rapid pacing rather than the limited perfusion-blood supply associated with coronary disease. ${ }^{34}$ The relevance of such a supplydemand mismatch as a basis for ischemic cardiomyopathy has been previously established, specifically in the porcine model, in which collateral vessel formation remains limited. ${ }^{13,14,35}$ Evidence supporting the use of this preparation as a relevant model of cardiomyopathy is further suggested in the present study by the blunted response to epinephrine observed in the AdNull-treated animals, which is consistent with the $\beta$-adrenergic downregulation characteristic of a cardiomyopathic state. ${ }^{13,14,36}$ Furthermore, demonstration of decreased ventricular function in naive animals paced without vector administration suggests that this effect is the direct result of pacing rather than a toxic effect of vector administration. Given these considerations, it seems plausible that angiogenic therapy is effective in this model because of its ability to enhance myocardial blood supply through increased vascularization.

In conclusion, we demonstrate that adenovirus-mediated transfer of VEGF 121 in a porcine model of myocardial dysfunction helps to preserve ventricular function and speed recovery of myocardial contractility after rapid ventricular pacing. This form of gene therapy may be useful for the treatment of ischemic cardiomyopathy clinically.

We thank Barbara Cushing for editorial assistance and Tony Davis for veterinary support.

\section{References}

1. Tavazzi L. Epidemiologic burden of heart failure. Heart. 1998; 79(suppl 2):56-9.

2. Massie BM, Parker M. Congestive heart failure: current controversies and future prospective. Am J Cardiol. 1990;66:429-30.

3. Gillum RF. Epidemiology of heart failure in the United States. Heart J. 1993;126:1042-7.

4. Brown TA. Hibernating myocardium. Am J Crit Care. 2001;10:84-91

5. Rinaldi CA, Hall RJ. Myocardial stunning and hibernation in clinical practice. Int J Clin Pract. 2000;54:659-64.

6. Wilson JM. Reversible congestive heart failure caused by myocardial hibernation. Tex Heart Inst J. 1999;26:19-27.

7. Thomas, KA. Vascular endothelial growth factor, a potent and selective angiogenic agent. J Biol Chem. 1996;271:603-6.

8. Mack CA, Patel SR, Schwarz EA, et al. Biological bypass with the use of adenovirus-mediated gene transfer of the complementary deoxyribonucleic acid for vascular endothelial growth factor 121 improves myocardial perfusion and function in the ischemic porcine heart. J Thorac Cardiovasc Surg. 1998;115:168-77.

9. Lee L, Patel S, Hackett N, et al. Focal angiogenic therapy using intramyocardial delivery of an adenovirus vector coding for endothelial growth factor 121. Ann Thorac Surg. 2000;69:14-24.

10. Giordano FJ, Ping P, McKirnan MD, et al. Intracoronary gene transfer of fibroblast growth factor-5 increases blood flow and contractile function in an ischemic region of the heart. Nat Med. 1996;2:534-9.

11. Tanaka E, Hattan N, Ando K, et al. Amelioration of microvascular myocardial ischemia by gene transfer of vascular endothelial growth factor in rabbits. J Thorac Cardiovasc Surg. 2000;120:720-8. 
12. Yang RH, Thomas GR, Bunting S, et al. Effects of vascular endothelial growth factor on hemodynamics and cardiac performance. J Cardiovasc Pharmacol. 1995;27:838-44.

13. Chow E, Woodard J, Farrar D. Rapid ventricular pacing in pigs: an experimental model of congestive heart failure. Am J Physiol. 1990; 258:H1603-5.

14. Kajstura J, Zhang X, Liu Y, et al. The cellular basis of pacing-induced dilated cardiomyopathy. Circulation. 1995;92:2306-17.

15. Rosenfeld MA, Yoshimura K, Trapnell BC, et al. In vivo transfer of the human cystic fibrosis transmembrane conductance regulator gene to airway epithelium. Cell. 1992;68:143-55.

16. Konstadt SN, Thys d, Mindich BP, et al. Validation of quantitative intraoperative transesophageal echocardiography. Anesthesiology. 1986;65:418-21.

17. Gorman JH 3rd, Gupta KB, Streicher JT, Gorman RC, Jackson BM, Ratcliffe MB, et al. Dynamic three-dimensional imaging of the mitral valve and left ventricle by rapid sonomicrometry array localization. $J$ Thorac Cardiovasc Surg. 1996;112:712-26.

18. Packer M, Carver JR, Rodeheffer RJ, et al. Effect of oral milrinone on mortality in severe chronic heart failure: the PROMISE Study research Group. N Engl J Med. 1991;325:1468-75.

19. Rosengart T, Patel SR, Crystal RG. Therapeutic angiogenesis: protein and gene therapy delivery strategies. J Cardiovasc Risk. 1999;8:534-9.

20. Lazarous DF, Shou M, Scheinowitz M, et al. Comparative effects of basic fibroblastic growth factor and vascular endothelial growth factor on coronary collateral development and the arterial response to injury. Circulation. 1996;94:1074-82.

21. Banai S, Jaklitsch MT, Shou M, et al. Angiogenesis-induced enhancement of collateral blood flow to ischemic myocardium by vascular endothelial growth factor in dogs. Circulation. 1994;89:2183-9.

22. Harada K, Friedman M, Lopez JJ, et al. Vascular endothelial growth factor administration in chronic myocardial ischemia. Am J Physiol. 1996;270:H17901-802.

23. Rosengart TK, Lee LY, Patel SR, et al. Angiogenesis gene therapy: phase I assessment of direct intramyocardial administration of an adenovirus vector expressing VEGF121 cDNA to individuals with clinically significant severe coronary artery disease. Circulation. 1999;100:468-74.

24. Rosengart TK, Lee LY, Patel SR, et al. Six-month assessment of a phase I trial of angiogenic gene therapy for the treatment of coronary artery disease using direct intramyocardial administration of an adenovirus vector expressing the VEGF121 cDNA. Ann Surg. 1999;230: 466-70.

25. Magovern CJ, Mack CA, Zhang J, Rosengart TK, Isom OW, Crystal RG. Regional angiogenesis induced in non-ischemic tissue by an adenovirus vector expressing vascular endothelial growth factor. Hum Gene Ther. 1997;8:215-27.

26. Losordo DW, Vale PR, Symes JF, et al. Gene therapy for myocardial angiogenesis: initial clinical results with direct myocardial injection of phVEGF 165 as sole therapy for myocardial ischemia. Circulation. 1998;98:2800-4.

27. Laham RJ, Selke FW, Edelman ER, et al. Local perivascular delivery of basic fibroblast growth factor in patients undergoing coronary bypass surgery: results of a phase I randomized, double-blind, placebo controlled trial. Circulation. 1999;100:1865-71.

28. Schumacher B, Pecher P, vonSpecht BU, et al. Induction of neoangiogenesis in ischemic myocardium by human growth factors: first clinical results of a new treatment of coronary artery disease. Circulation. 1998;97:645-50

29. Udelson JE, Dilsizian V, Laham RJ, et al. Therapeutic angiogenesis with recombinant fibroblast growth factor- 2 improves stress and rest myocardial perfusion abnormalities in patients with severe symptomatic chronic coronary artery disease. Circulation. 2000; 102:1605-10

30. Hendel RC, Henry TD, Rocha-Singh K, et al. Effect of intracoronary recombinant human vascular endothelial growth factor on myocardia perfusion: evidence for a dose-dependent effect. Circulation. 2000; 101:118-21.

31. McKirnan MD, Guo X, Waldman L, et al. Intracoronary gene transfer of fibroblast growth factor-4 increases regional contractile function and responsiveness to adrenergic stimulation in heart failure. Card Vasc Regen. 2000;1:11-21.

32. Magovern CJ, Mack CA, Zhang J, et al. Direct in vivo gene transfer to canine myocardium using a replication deficient adenovirus vector. Ann Thorac Surg. 1996;62:425-33.

33. Safi J, DiPaula A, Riccioni T, et al. Adenovirus-mediated acidic fibroblastic growth factor gene transfer induces angiogenesis in the nonischemic rabbit heart. Microvasc Res. 1999;58:238-49.

34. Vogt Am, Kubler W. Heart failure; is there an energy deficit contributing to contractile dysfunction? Basic Res Cardiol. 1998;93:1-10.

35. Roth DA, Urasawa K, Helmer GA, Hammond KH. Downregulation of cardiac guanosine 5'-triphosphate-binding proteins in right atrium and left ventricle in pacing-induced congestive heart failure. J Clin Invest. 1993;91:939-49.

36. Marzo KP, Frey MJ, Wilson JR, et al. $\beta$-Adrenergic receptor-G protein adenylate cyclase complex in experimental canine congestive heart failure produced by rapid ventricular pacing. Circ Res. 1991;69:1546-56.

\section{Availability of Journal back issues}

As a service to our subscribers, copies of back issues of The Journal of Thoracic and Cardiovascular Surgery for the preceding 5 years are maintained and are available for purchase from Mosby until inventory is depleted. Please write to Mosby, Subscription Customer Service, 6277 Sea Harbor Dr, Orlando, FL 32877, or call $800-654-2452$ or $407-345-4000$ for information on availability of particular issues and prices. 